\title{
COVID-19 and information - communication technology: Common components in an interactive framework for predicting, preventing, controlling and monitoring the new COVID-19 disease
}

\author{
Azita Yazdani \\ Shiraz University of Medical Sciences \\ Roxana Sharifian \\ Shiraz University of Medical Sciences \\ Ramin Ravangard \\ Shiraz University of Medical Sciences \\ seyedeh fatemeh dashti \\ Bushehr University of Medical Sciences \\ Marzieh Shukohifar \\ Shahid Sadoughi University of Medical Sciences \\ Yousef Gholampour \\ Fasa University of Medical Science \\ maryam zahmatkeshan ( $\nabla$ mokatebatedari2020@gmail.com) \\ Fasa University of Medical Science https://orcid.org/0000-0003-4090-391X
}

\section{Research article}

Keywords: Epidemic, Outbreak, Disease control, Medical informatics, E-health, Conceptual model, Tele-monitoring, Internet of things, Fog computing, COVID-19, New corona viruses

Posted Date: September 29th, 2020

DOI: https://doi.org/10.21203/rs.3.rs-49919/v1

License: (a) (1) This work is licensed under a Creative Commons Attribution 4.0 International License. Read Full License 


\section{Abstract}

Background

Since there is no specific treatment for coronavirus, there is an urgent need for global monitoring of people with Covid-19. The use of e-health services should be compatible with the diagnosis and control of the outbreak of zoonotic infectious diseases. The aim of this study is to provide a conceptual model based on health information technology services for Covid-19 disease management.

\section{Methods}

The present study is an applied descriptive study that was performed on a cross-sectional basis in a COVID-19 Center Hospital in Fars province of IRAN country in April 2020. The main tool of this research is a questionnaire that has been compiled by reviewing related articles in databases and surveying with experts in order to determine the necessary services in the management and control model of the prevalence of Covid19 disease. Then, in order to determine the necessary services in the conceptual model, this questionnaire was given to various specialists in the COVID-19 Center Hospital. Finally, based on the results of the questionnaire, a comprehensive conceptual model for the management and control of COVID 19 diseases is presented.

Results

The proposed model consisted of three layers of cloud computing, fog and data acquisition. All services were approved by the surveyed participants. Among the services, Tele- monitoring for home quarantine, Electronic self-assessment, Telepsychology of patients in home and hospital quarantine, Tele prescription, Tele- information Tele- training have the highest agreement rate. proposed model is an integrated model. The innovation that can be mentioned in this research is the use of priority queue service as a service of the fog layer.

Conclusion

Information communication technology tools have an important role in all aspects of contagious diseases management.

\section{Background:}

- Introduction: Information and Communication Technology (ICT), including the internet, wireless networks, mobile phones, cloud computing, fog computing, and the Internet of Things (IoT), are used as health care support services [1]. Given the role of healthy citizens in the development of any country, governments allocate significant funding for health information technology infrastructure [2]. According to the World Health Organization's report on managing epidemics in 2018 , the 21 st century has already been marked by major epidemics. The epidemics in this century are spreading faster and further than ever. Outbreaks that were localized in the past can now become a global issue very quickly, in fact, as fast as an intercontinental aircraft can fly [3]. Infectious diseases are both a national threat to countries and a potential international crisis. Continuous monitoring of the prevalence of airborne, contagious and deadly diseases is the most important part of health care support services [1, 4]. If governments or health insurance companies can monitor healthcare utilization on a daily basis and spread of infectious diseases at national level in real time, a great opportunity will be provided to manage health care services. Healthcare policymakers and insurance company managers can predict the best strategy to effectively manage serious illnesses by monitoring the use of health care services and illnesses [5]. Monitoring the use of healthcare utilization and the occurrence of diseases on a daily basis and at the national level in real time is vital. Healthcare Utilization Monitoring System (HUMS), which was introduced in 2015 , is one of the most successful systems in using information technology to monitor and identify the use of health care services. The launch of such systems could certainly help governments to act in a timely manner to prevent the spread of infectious diseases and prevent secondary infections during the outbreak of infectious diseases such as MERS [5]. During epidemics, diagnosis can only be made on the basis of symptoms; this is while early diagnosis is necessary both for treatment and prevention of infectious diseases [7]. Monitoring the health of people in the community plays an important role in controlling and preventing the spread of infectious diseases. For example, continuous monitoring of the respiratory system can severe acute respiratory syndrome (SARS) [8]. Studies show that patient self-care and tele-monitoring reduce the number of hospital visits and can also predict the likelihood of getting the disease [9]. In the treatment of an epidemic such as influenza, time is greatly important. In annual epidemic diseases, telemedicine allows rapid and appropriate use of treatment and limits general exposure to infected individuals [7]. The use of telemedicine in epidemic conditions has the potential to improve epidemiological research, disease control, and clinical management [10]. Information and communication technology and mathematical-based models have been widely used for the prevention and early prognosis of deadly epidemics [4]. Outbreak modeling can also help us to understand the ways that a disease speeds, and as a result, it enables us to find the best ways to prevent the spread and fight against infectious viral diseases [11]. Corona viruses (CoV) are a large family of infectious viruses that can lead to a variety of illnesses, from colds to more severe diseases such as Middle East Respiratory Syndrome (MERS$\mathrm{CoV}$ ) and Sever Acute Respiratory Syndrome (SARS-CoV). The new coronavirus (nCoV) is a new strain of this virus that has not been previously detected in humans. The new coronavirus first appeared in Wuhan, China, on December 19, 2019, and spread rapidly in other countries. Researchers in this short period of time have been looking at various aspects of this unknown virus. Most articles published since the outbreak of coronavirus until April 2020 have been focusing on various aspects, including the characteristics and lessons learned from Covid-19 [14], epidemics [15, 16], pathology [15], descriptive and exploratory analysis of identified cases [17], the role of radiology in rapid diagnosis, prognosis and control [18-21], the effect of chloroquine phosphate in treatment [22], the role of alternative methods in controlling the prevalence [23], comparison of three groups of corona viruses such as SARS, MERS and novel FI [24], and pregnancy care in coronavirus [25]. Due to the rapid spread of new coronavirus 19 in many countries and regions, the number of patients with the virus has exceeded the capacity of hospitals. Healthcare professionals working to manage this 
unprecedented crisis are faced with the question of what is the best way to coordinate medical resources used in completely separate locations. Due to the high risk of infection and the characteristics of human-to-human transmission, many cities are being locked down. As a result, not only the patients have been marginalized, but also many physicians working in regional hospitals have been having limited access to the necessary counseling and treatment guidelines from provincial hospitals to manage cases of Covid-19 pneumonia. As long as this crisis persists, relying solely on traditional communication practices, such as a doctor's visit or face-to-face counseling on a health care professional network, can lead to significant costs and increased health concerns. Taking advantage of telehealth paradigms, including telemedicine, some countries have succeeded in combating the epidemic by combining information and communication technology with health-related services. Combining online consultation and real-time clinical data exchange can remotely support the digitalization of workflow. Due to the novelty of Covid-19 disease, articles that have examined the role and use of information and communication technology in controlling, preventing and predicting the disease until March 2020 are very few $[27,28]$. Moreover, these limited studies have mainly focused on the pathology and treatment of this disease. The aim of this study is to provide a conceptual model based on information and communication technology in order to effectively manage the Covid-19 disease.

\section{- Related work:}

The application of information technology in the prevention, control and management of infectious diseases that have occurred in recent years has been discussed in this section. Technologies have been examined in three general categories:

- Electronic health (e-health)

- Cloud computing, fog and the internet of things (IoT)

- Data mining

\section{- Electronic health:}

Electronic health is an emerging field in the medical informatics, public health and business, which enables health services and information to be distributed or enhanced through the internet and related technologies. In the e-health, information communication technology (ICT) is used for improving and delivering health services and related information. Telemedicine, Telehealth, Mobile-health, Mobile patient monitoring are examples of e-health. Telemedicine is a commonly used term in the healthcare sector and is a type of e-health $[29,30]$. Telemedicine is often seen as a way to improve the management of chronic diseases and help medical emergencies [30-32]. Research shows that the use of this technology during epidemics has a high potential in disease control [32]. telemedicine applications in epidemic conditions can be divided into 6 conditions:

1: teleconsultation for affected patients who are quarantined in health centers or at home.

2: Tele-expertise that enables the exchange of views between healthcare team.

3: Maintaining the care of patients, who are unable to access health care facilities.

4: teleconsultation for healthy people who are quarantined at home.

5: Tele-monitoring to identify people suspected of having the disease.

6: Tele-radiology

The launch of remote counseling systems, while reducing unnecessary referrals to infectious disease health centers to assess health status, reduces the transmission of disease to healthy individuals [33]. Therefore, remote medical counseling prevents healthy people who are quarantined at home from going to care centers when they are suspected of having the disease. It also helps medical team to visit people at their home for further examination. Among studies done in this area we can point to tele- counseling through a mobile-based telemedicine application to identify suspected cases of Ebola during the outbreak of Ebola in West Africa. An analysis of online questionnaires that collect data on the health status of people in the community at the time of outbreak of a contagious disease allows health monitoring centers to identify people suspected of having the disease through a tele-monitoring system. Other examples of telemonitoring used during the EVD epidemic include the use of smartphone in Sierra Leone for Contact Tracing [34, 35], as well as the integrated platform based on open data kit and form hub technology in Nigeria [36]. In 2014, a mobile application for telemonitoring was developed by the United Nations Population Fund with the outbreak of Ebola in Guinea. "Ebola Tracks" was a text message-based remote monitoring system that was used in 2014 in Western Australia to monitor people returning from African countries affected by Ebola. The health status of those who were potentially exposed to the virus was checked twice a day via text message. One of the parameters used in this system was body temperature [37]. In order to reduce the time that care providers spend on communicating with patients quarantined at home or in medical centers, tele counseling has been considered as an acceptable solution. This system shortens the chain of infection and the virus. During the 2003 epidemic of Acute Respiratory Syndrome (SARS) in Taiwan [38], the 2009 H1N1 flu pandemic [38], the 2013 H7N9 flu in China [39], and the 2014 Ebola care in the United States, teleconsultation was used to assess the patients' status. In 2015 coronavirus epidemic (MERS), South Korea's care centers used tele-prescription to prescribe medication for people who were in quarantine [10]. In regard to the application of telemedicine at the time of epidemic, we can refer to the tele-expertise or distant experience that enables the physicians in a region to benefit from the experiences and expertise of their colleagues [41]. This system helps them to communicate with each other and provide multidisciplinary care. Also, when local medical centers do not have a clinical specialist to diagnose the disease or treat patients, the main supportive centers can advise their colleagues remotely [42]. Protecting the mental health of employees is essential for 
better control of infectious diseases, but the best approach in this area during epidemics is unknown [41]. In order to respond to the psychological pressures of employees during the outbreak of infectious diseases, there is a strong need for psychological counseling in medical staff [43]. Using a tele psychological system is an effective solution for this problem. It is also essential to create a psychological intervention team, which provides online courses to guide medical staff to deal with common psychological problems. In addition to medical staff, psychological skills are needed to deal with the anxiety, fear and other emotional problems of quarantined patients [44]. tele-radiology is a branch of telemedicine in which, information and communication technology is used to transmit radiological images from one place to another. The goal of this service is to share images with other radiologists and physicians in order to enable remote diagnosis, consultation and interpretation of radiological images. The outbreak of pneumonia, such as SARS and bird flu, highlighted the importance of tele -radiology system. For example, during the outbreak of SARS, many countries formed a national panel of specialists to determine case status and examine clinical, radiological, and epidemiological data from suspected patients [45]. Tele-radiology services eliminate the need for transferring patients to crowded hospitals and provide real-time diagnostic counseling by specialists located across the country and the world to meet the needs of local specialists [42]. Studies show that chest CT has a low misdiagnosis rate in COVID-19 diagnosis and may serve as a standard method for rapid diagnosis of COVID-19 to optimize patient management. Given the critical conditions that have plagued the world with the spread of infectious diseases, the use of tele-radiology services can lead to the rapid and effective transfer of findings to physicians [46]. In a study [42] using the WhatsApp messenger platform, tele-radiology services were used in order to diagnose Covid-19 in Iran. In this study, the authors used social media to connect volunteered physicians in North America and committed physicians in Iran for tele-radiological counseling [42]. This communication strategy allows radiologists from around the world to provide advice for specialists in areas with limited access to chest radiology during a rapid expanding epidemic.

\section{- Data mining:}

Computers and data mining techniques help to analyze, diagnose, predict and control diseases caused by viral infections. By performing various data mining methods on data collected in infectious disease control centers, it is possible to better understand the factors that affect the occurrence of infection and the possibility of recovery from various infectious diseases [47]. Infections such as MERS-CoV spread easily and they have high mortality rate. Therefore, the establishment of predictive systems and the diagnosis of these diseases with a high degree of accuracy can reduce the prevalence and mortality rate of these diseases [47]. Studies show that early detection of MERS-CoV infection can help to control the spread of virus and reduce human suffering [48]. In recent years, researchers have used various machine learning classification techniques on MERS-CoV datasets. In a study [47], data mining models were developed using decision tree classification algorithms, Naive Bayes and J48 to predict stability and improve MERS-CoV infection. This study used the control and command center data set of Saudi Ministry of Health to build the model. The accuracy of this model was estimated between $53.6 \%$ and $71.58 \%$. Researchers in a study [49] proposed a cloud-based MERS-CoV prediction system. This system is based on Bayesian Belief Networks (BBN) for primary classification of patients. The researchers used a geo-location system to show patients on Google Maps. Patients who were infected could be tracked by GPS of their mobile phones. This proposed system is useful for citizens as it allows them not to enter the contaminated areas. In addition, health officials can effectively manage the problem of infection. The result of this study allowed the prediction of MERSCoV-infected areas in Google maps with high classification accuracy. In a study [48], to establish a disease prediction model, the Support Vector Machine (SVM), Decision Tree, and K-Nearest Neighbor (K-NN) classifications were used on the MERS-CoV dataset, which included all reported cases in Saudi Arabia between 2013 and 2017. In a study [50], the researchers used three data mining algorithms of Apriori, Decision Tree, and SVM to compare and differentiate between two viruses with similar symptoms to SARS CoV and MERS CoV. They used the data of spike glycoprotein from NCBI. Results of the study distinguished the MERS and SARS spike glycoprotein with high accuracy. A study [51] used data mining techniques in a text-based MERS-CoV dataset to identify high-precision binary, multi-class, and multi-label classification models. The dataset included all MERS-CoV cases in Saudi Arabia that were collected from the Saudi Ministry of Health from 2013 to the second half of 2016. The results of this study showed that the decision tree is the most accurate algorithm for classifying binary classes, while k-NN is the most accurate algorithm for classifying multiple classes. In 2016, Kerdprasop used remote assessment data to model the main characteristics of Deng's outbreak with regression and binning classification. The proposed model achieved 91-95\% accuracy [52]. Different decision support systems have been proposed to prevent the spread of H1N1. In 2015, Lai et al [53] developed a system to predict H1N1 pandemic based on daily influenza cases and general population in Hong Kong. They used spatio-temporal and stochastic SEIR (stsSEIR) model to predict the number of cases for the next two days. In 2014, Dias and Arruda proposed a mathematical based cost optimization model to control the H1N1 epidemic based on the limited cost optimization model [54]. In 2014, Farah et al [55] developed a dynamic epidemic model for H1N1 Influenza using a Bayesian -based simulator. One of the most effective ways to prevent and control the spread of disease is to monitor and follow the news and social media about the spread of infectious diseases. Today, large amounts of emergency and health data are increasingly being obtained from a wide range of websites and social media. This information can be very useful for monitoring the disease and early detection of its outbreak [56]. Due to the growth and development of social networks, the use of web news mining techniques can identify the demographic and geographical information of users with high accuracy [27]. This is only effective if these networks report statistical data along with the related comments, photos, and videos about the infectious diseases. This leads to the prediction of mortality rate in each region, and raises the attention of policymakers to support health care systems in these areas in order to implement targeted training programs in the areas, which ultimately reduce the incidence and mortality rate in the societies [27]. In recent years, several public web monitoring projects have been introduced to monitor and detect early outbreaks of infectious diseases, which include GPHIN [57], BioCaster [58], HealthMap [59], EpiSpider [60], MedISys [61], and Google Flu Trends [62]. The growing popularity of blogs, micro-blogs, and social networks has recently become a valuable new source of information for monitoring disease outbreaks. Among the articles that have shown the potential of Twitter messages to detect and predict the outbreak of disease, we can point to the articles [63] that predicted a swine flu pandemic, [64, 65] detected influenza epidemics, [66] used surveillance system to detect influenza and cancer and [67], all of which have used different machine learning techniques to predict and diagnose the prevalence and classify patients. Recently, a study [27] used the FAMEC method to send a warning message to monitoring systems in order to detect quarantined medical centers prevalence in a timely manner. In the model presented in this study, unstructured data

Page $4 / 15$ 
on coronavirus (2019-nCoV) were extracted from Twitter and after the text cleaning processes, they were classified. All of the systems and solutions that have been presented demonstrate the successful use of machine learning techniques to extract and acquire new knowledge for public health care purposes.

\section{- Cloud computing, fog and internet of things (loT):}

The industry 4.0 and its main information and communication technologies are completely changing services and the world of production. This is especially true in the area of health care, where the loT, cloud computing and fog, as well as Big Data technologies are changing the e-health and its entire ecosystem, leading it to health 4.0. Hospitals need high-capacity information technology infrastructure for patients' electronic health records and data related to personal, patients and physicians [68]. Storing this data helps to prevent the spread of infectious diseases. The most important part of health care support services is regular monitoring of airborne disease outbreaks [1]. Smart sensors and devices used to monitor the patient's health have computational limitations and limited storage capacity, so they are not able to cope with the large amount of generated data [69]. Cloud computing approach has been adopted to address such challenges. The cloud computing model provides the ability to store, process, and manage large volumes of data [70]. It actually provides high data processing capabilities, along with no storage limitations. Cloud computing, advances in mobile phone technology, and wearable wireless sensors have motivated cloud-based healthcare services. Cloud computing is a paradigm that provides information technology (IT) resources over the internet. Using cloud computing, a huge amount of virus-related data can be linked through social media and other health-related web services [1]. The use of cloud computing in the provision of healthcare support services also enables people to manage their health effectively. The modern healthcare industry is unpredictable without integration with internet-based information technology. The loT devices such as wearable sensors, environmental sensors, etc. collect significant data on patient health care, which must be processed efficiently [1]. By combining sensors, information technology, artificial intelligence, and networking devices, the loT can lead to communication between hospitals, patients, and telemedicine devices, which improve medical conditions. Studies show that loT is useful in controlling and tracking sever acute respiratory infection [71]. Combination of the Internet of Things (IoT) and related technologies can play an important role in preventing the spread of zoonotic infectious diseases [24]. During the outbreak of infectious diseases, the disease control centers need to monitor specific areas. Many efforts have been made to develop portable, user-friendly and cost-effective systems to make diagnosis in the pint of care. In a paper [72], an loT system has been introduced that can become an essential tool for health care centers to combat the spread of infectious diseases that have been identified by DNA or Ribonucleic acid (RNA). Despite the benefits of cloud computing, due to its centralized nature, it is unable to provide services with minimal latency, location awareness, and geographic distribution that are essential for loT applications. Since the cloud computing approach requires constant internet connection at a reliable speed with sufficient bandwidth, in cases where bandwidth is insufficient and network interruptions occur, cloud delays would increase and would be difficult to provide healthcare services [73]. In order to provide health care services where decision-making and response to events with minimal delay is necessary [74], another model of processing called fog computing is used. Fog computing is in fact a generalization of cloud computing. Therefore, fog calculations can provide immediate solutions in catastrophic or epidemic cases and provide a platform for monitoring health status, diagnosis and treatment to prevent the spread of life-threatening diseases. A limited number of studies have investigated the benefits of fog computing in the process of managing infectious diseases in their proposed models. In a study [75] a health monitoring system based on fog computing has been proposed for realtime monitoring and analysis of people's health, statistics and related events such as health data, location-based data, drug data, environmental data and meteorological data to control chikungunya virus. Accumulated data from various events are integrated into a common template and will be sent to the fog layer instead of cloud layer for fast processing and high accuracy. In the fog layer, the Fuzzy-C means technique is used to categorize the user into two categories of infected or uninfected based on the collected symptoms. After a person is diagnosed with the virus, an alert message will be sent to the infected person, health care team, and non-infected people as well as those who have visited danger areas or live in hazardous or contaminated areas to take precautions and immediate action to prevent the spread of CHV. The results of this study confirmed the benefits of fog layer alongside the cloud layer to achieve faster detection, reduce latency, and optimize bandwidth usage. A study [1] provided a framework based on fog architecture that categorized dengue patients into three categories of uninfected, infected, and severely infected using a dataset built in 2010 . The purpose of this proposed framework was to create a latency-aware system to categorize users into different categories based on related symptoms using loT sensors and audio/video files. To achieve this, a smart framework consisting of three components has been proposed, which include the loT layer, the fog infrastructure, and cloud computing. By the use of network devices within the fog infrastructure, the system delay time is reduced. The data generated by the loT layer is first processed by the devices in fog layers, which are at close distance with the user. The raw data and generated data are then stored in cloud infrastructure and then, they are sent to various institutions, including the user, hospital, physician, and public health organizations. Experimental evaluation of this hypothesis proved that using fog infrastructure can provide a better response time for delay-sensitive programs with the least impact on system accuracy. Article [4] provides an effective cloud computing architecture that predicts patients infected with $\mathrm{H} 1 \mathrm{~N} 1$ and provides preventive measures for infection control. This program includes four processing components along with a secure medical database for cloud storage. The random decision tree is used for initial evaluation of the infection in each patient depending on his/her symptoms. Social Network Analysis (SNA) is used to present the outbreak situation. Other studies that have proposed cloud computing architectures at the time of outbreak of infectious diseases include; Tazkia et al (2015) study that attempted to identify the possible prevalence of dengue virus based on statistical calculations and geographic information system (GIS) [76], Sandhu et al (2016) study [4] that aimed to prevent and predict H1N1 influenza and MERS-Corona, and a study [77] that proposed a loTbased cloud framework to control the outbreak of Ebola virus with continuous tele-monitoring of infected patients in real time using radio frequency identification (RFID) technology, cloud computing and J48 decision tree. Since there is no specific treatment for coronavirus, there is an urgent need for global monitoring of those infected with Covid-19 [24]. In a study [28], for early detection and rapid improvement of Covid-19, the web-based program was presented for intelligence diagnosis under the name of nCapp. This intelligent system classifies patients in mild, moderate, severe, or critical pneumonia. This cloud based system has the ability to update its database online and update its detection model based on the latest data. In connection with infectious diseases, remote detection and monitoring of infections is vital for controlling the spread of disease in real time. In the event of an outbreak of

Page 5/15 
infectious disease, people in the community who go to medical centers can be at risk of getting the virus [75]. Therefore, the cloud - fog computing, which is based on loT tools and clinical decision-making systems, is an important step in early detection of infectious diseases, reducing referrals to health care centers and ultimately, reducing the risk of infection in the community.

\section{Methods:}

The literature on contagious disease management based on information-communication technology (ICT) was searched on Medline, Scopus, Science

Direct, Web of Science, OVID, Springer, Google Scholar, Elsevier, and ProQuest using "Infectious Diseases" and "E-health" paradigm such as "Telemedicine", "Tele-consultation", "Mobile health", "Tele-radiology", and "Industry 4.0 and its main information and communication technologies" such as Internet of Things, Cloud and Fog Computing, and data mining combined with specific contagious disease (e.g.:MERS, EBOLA, SARS, Covid-19, Influenza etc.). Article reference lists and websites of public health departments, WHO, and disease-specific organizations were also searched. Selected articles and literature were related to contagious disease management based on ICT, description of program implementation, and health policy issues regarding contagious disease management programs. The search was limited to English articles and excluded dissertations. The authors reviewed each abstract to eliminate irrelevant articles. Selected articles were reviewed to identify services and elements of contagious disease management based on ICT. The present study is an applied descriptive study that was performed on a cross-sectional basis in a Corona Center Hospital in Fars province of IRAN country in April 2020. The study population consisted of all physicians, nurses, laboratory staff, and specialists in the field of medical informatics, health information management and Healthcare Services Management, which numbered over 700 people. With the help of Morgan's Sampling Table and using classified cluster sampling, 250 people were examined, including doctors (40 people), nurses (160 people), laboratory staff ( 25 people), and medical informatics and health information management and Healthcare Services Management (35 people).people were selected separately and randomly. The main tool of this research is a questionnaire that has been compiled by reviewing related articles in databases and surveying with experts in order to determine the necessary services in the management and control model of the prevalence of Covid19 disease. The validity of the questionnaire was confirmed by experts and its reliability was based on a preliminary sample with the distribution of the questionnaire among 40 people and was confirmed by Cronbach's alpha coefficient of 0.89 . Then, in order to determine the necessary services in the conceptual model, this questionnaire was given to 250 specialists from a team of clinical specialists, medical informatics, health information management, and Healthcare Services Management. For the questionnaire axes, 5-point Likert scale was made. The response scales ware : 1 = Strongly Disagree, $2=$ Disagree, $3=$ Neutral, $4=$ Agree, $5=$ Strongly Agree. Also, at the end of the questionnaire, there was an empty space for registering the services offered by the panel members, user comments about its benefits, problems and limitations. The criterion for considering services was the collective agreement to reach a level of $75 \%$ or 3.75 and above of the 5 participants regarding the "agreement" rate. The sum of the two scales of "agree" and "strongly agree" is considered the "agreement" rate. Excel software was used to provide descriptive statistics. Finally, based on the results of the questionnaire, a comprehensive conceptual model for the management and control of COVID 19 diseases is presented. In order to draw this conceptual model, we used Microsoft Visio software.

\section{Results:}

Table 1 shows the results of questionnaire evaluation. According to the results of this table, more than $75 \%$ agreement rate was reached for all services, so Delphi was done in one stage. 


\begin{tabular}{|c|c|c|c|c|c|}
\hline \multirow[t]{2}{*}{ Service Description } & \multirow{2}{*}{$\begin{array}{l}\text { Strongly } \\
\text { Disagree }\end{array}$} & \multirow[t]{2}{*}{ Disagree } & \multirow[t]{2}{*}{ Neutral } & \multicolumn{2}{|c|}{ Agreement rate $(\mathrm{N}) \%$} \\
\hline & & & & Agree & $\begin{array}{l}\text { Strongly } \\
\text { Agree }\end{array}$ \\
\hline $\begin{array}{l}\text { Tele- monitoring of the health status of patients with the new coronavirus who are } \\
\text { under home quarantine }\end{array}$ & $0 \%$ & $0 \%$ & $0 \%$ & $(0) 0 \%$ & $(250) 100 \%$ \\
\hline $\begin{array}{l}\text { Tele-monitoring of the health status of patients with the new coronavirus who are } \\
\text { hospitalized }\end{array}$ & $0 \%$ & $0 \%$ & $0 \%$ & (7) $2.8 \%$ & (243) $97.2 \%$ \\
\hline $\begin{array}{l}\text { Electronic self-assessment of general population to assess the status of new } \\
\text { coronavirus infection }\end{array}$ & $0 \%$ & $0 \%$ & $0 \%$ & $(0) 0 \%$ & $(250) 100 \%$ \\
\hline Telepsychology of hospital staff who care for patients with new coronavirus & $0 \%$ & $0 \%$ & $0 \%$ & $(8) 3.2 \%$ & (242) $96.8 \%$ \\
\hline Telepsychology of patients with new coronavirus who are under home quarantine & $0 \%$ & $0 \%$ & $0 \%$ & $(0) 0 \%$ & $(250) 100 \%$ \\
\hline Telepsychology of patients with the new coronavirus who are hospitalized & $0 \%$ & $0 \%$ & $0 \%$ & $(0) 0 \%$ & (250) $100 \%$ \\
\hline $\begin{array}{l}\text { Tele multiple disciplinary care between physicians to share experiences and provide } \\
\text { multidisciplinary care to patients with the new coronavirus }\end{array}$ & $0 \%$ & $0 \%$ & $0 \%$ & $(16) 6.4 \%$ & (234)93.6\% \\
\hline $\begin{array}{l}\text { Tele-radiology to interpret the results of chest CT scans of patients with the new } \\
\text { coronavirus }\end{array}$ & $0 \%$ & $0 \%$ & $0 \%$ & (5) $2 . \%$ & $(245) 98 \%$ \\
\hline $\begin{array}{l}\text { Radiological imaging processing service by artificial intelligence for precise and early } \\
\text { diagnosis of new coronavirus }\end{array}$ & $0 \%$ & $0 \%$ & $0 \%$ & $(50) 20 \%$ & $(200) 80 \%$ \\
\hline $\begin{array}{l}\text { BIG Data analysis for various goals such as Prediction of the survival time of } \\
\text { hospitalized patients with the new coronavirus based on the analysis of data } \\
\text { collected from patients }\end{array}$ & $0 \%$ & $0 \%$ & $0 \%$ & (27) $10.08 \%$ & (223)89.2\% \\
\hline $\begin{array}{l}\text { Tracking the location of patients with the new coronavirus to identify suspected sites } \\
\text { infected with the new coronavirus }\end{array}$ & $0 \%$ & $0 \%$ & $0 \%$ & $(0) 0 \%$ & $(250) 100 \%$ \\
\hline $\begin{array}{l}\text { Tele prescription service for patients with new coronavirus who are under home } \\
\text { quarantine }\end{array}$ & $0 \%$ & $0 \%$ & $0 \%$ & $(24) 9.6 \%$ & (226) $90.4 \%$ \\
\hline $\begin{array}{l}\text { Tele information service regarding the news of the new coronavirus (prevention, } \\
\text { treatment, prevalence and mortality, etc.) }\end{array}$ & $0 \%$ & $0 \%$ & $0 \%$ & $(0) 0 \%$ & $(250) 100 \%$ \\
\hline Tele- training service for the general public and medical staff & $0 \%$ & $0 \%$ & $0 \%$ & $(0) 0 \%$ & $(250) 100 \%$ \\
\hline
\end{tabular}

All of these services have been used in the proposed framework. Also, $2 \%$ participants in the survey commented on the suggestions section, all of whom were emphasizing on the need for the integration of these services in a comprehensive platform.

\section{Proposed conceptual model:}

Figure 1 shows the proposed conceptual model for effective management of the new coronavirus. In the proposed framework, loT technologies, fog and cloud computing, and queuing networks have been used to create a comprehensive platform for the management of COVID-19 diseases. The layering approach in the proposed model consisted of three layers of data acquisition layer, fog layer, and clod layer. Different databases such as EHR, GIS, and PACS(Picture Archiving and Communication Systems) allocated to the cloud layer. Different services use these databases. (Figure 2).

Figure 1. Proposed conceptual model

The data collection, preprocessing, include of encryption, compression, filtering, data standardization, data fusion, and data management services, fog update are commonly used in cloud and fog-based architectures. In the model presented in this study, in addition to the above-mentioned services, 14 new effective management services for the new corona virus were added based on the results of questionnaire. Each of these services has its own defined essential function, which is used as an input to adjacent services.

\section{A: Data acquisition layer:}

In this layer, data related to COVID 19 virus and data related to the environment and location of people are widely obtained from various loT devices embedded in different places at home and hospital, as well as sensor network embedded on the patient's body. Each sensor node is integrated with health sensors, location sensors, and environmental sensors. This information is sent to the fog layer for processing (Figure 2).

Figure 2. Three layers model

\section{B: Fog layer:}


The fog layer (Figure 3) preprocessing, networking, storage, encryption, compression, filtering, data standardization, data fusion, and queue management. The data sent from the data acquisition layer is stored in this layer. This storage is necessary for local processing in the fog layer. Another operation performed on data stored in the fog layer is data encryption with the aim of increasing privacy. Another fog layer service is standardization of data based on various medical informatics standards. Data filtering service in this layer cleans the data before analysis based on various filtering and noise elimination techniques. Data fusion service is used to combine collected data, delete additional data, replace new data and reduce the volume of collected data. Data analysis service in the fog layer processes data locally. Compression methods are used to reduce the amount of data that is transmitted over the network from the fog layer to the cloud layer. In the proposed conceptual model, in addition to the services mentioned in the fog layer, to increase the speed of data transfer between the fog and cloud layers, reduce the bandwidth used to send data to the cloud and reduce the response time to data collected by Covid-19 patients for health monitoring, 2 more services have been added:

\section{- Event classification service:}

It is for Through this service, the patient's condition is classified in three classes based on the data received, which include low risk, medium risk, and high risk. In the high risk cases, the data received from the patient is out of normal range and the person has underlying diseases or other risk factors. In the medium risk cases, some patient data is out of normal range and does not have underlying disease or other risk factors. In low risk cases, the patient's parameters are not outside the normal range. For classifying patient data into two classes of medium risk and high risk, the range of normal values, history of underlying diseases, or other risk factors are considered as thresholds. The total patient data sent is considered an event. In this way, the event will be sent to the cloud for review and decision making only if the threshold is violated, and a warning message will be sent to the health care team.

\section{- Queueing service:}

The task of this service is to prioritize the events sent by each patient and transfer them to the cloud layer and make the final decision. This service is responsible for sending events that have one or more parameters out of the normal range to be sent to the cloud. However, as the number of these events increases, a queue of events that need to be sent to cloud will be formed. The task of priority queue service is to prioritize the events according to the severity of patient's condition and to place them in the queue for sending. The type of underlying disease, or other risk factors and also the criticality of abnormal parameters play a major role in the prioritization of events.

Figure 3. The fog layer details

\section{Cloud layer:}

most of the services offered by the proposed model for managing and controlling the prevalence of Covid-19 are in this layer.

- E-Self-assessment service: Using this service, the general public can evaluate their health status by answering questions related to the diagnosis of new coronavirus. The self-assessment service also allows the screening to identify people suspected of having the disease. This service is a good alternative for people who go to medical centers and prevent any congestion that helps coronavirus to spread.

- Tele-information and Tele-training services: These services are a responsive system that includes the preparation and dissemination of reliable information and training on the new coronavirus. The audience of these services is the general public and medical staff. With these two services, the guidelines and instructions of healthcare staff are available for this group of users. Educational content (video, text, etc.) can be provided for the general public in the field of prevention, care, and treatment. Educational content for high-risk groups (people with cancer, diabetes, etc.) can also be provided by Tele-training services. Audience from both services will be able to receive the latest news about the outbreak, the number of people living with the disease and the number of deaths from Covid-19.

- Telepsychology service: This service provides remote psychological counseling for:

$$
\begin{aligned}
& \text { - Patients } \\
& \text { - medical staff } \\
& \text { - the general public }
\end{aligned}
$$

In order to manage their stress. Psychological counselors provide multimedia counseling services (voice, image, text) for hospitalized patients, patients in home quarantine, healthcare staff, and healthy people in home quarantine at regular intervals or on request. Therefore, mental health employees, while maintaining their health, can directly consult different target groups through this service.

- Tele-monitoring service: This service is for patients in

- The intensive care unit(hospital quarantine)

- home quarantine.

The purpose of this service is to provide tele-monitoring of the health status of patients by the medical staff. After the patient's information is recorded by the hospital, the patient's condition is monitored at home to prevent recurrence of the disease at the shortest time possible. The data of hospitalized patients are collected by loT devices. Patients at home also measure and record their symptoms during standard follow-up scheduling periods, as 
determined by the care center. This data is also collected by loT devices. The collected data will be evaluated in the fog layer by the classification service and will be sent to the cloud layer if the event is at high risk or medium risk category. Events are sent to cloud-based decision-making systems for further review. Evaluation results along with patient data are provided to the healthcare team and then, the treatment staff can take the necessary measure or action depending on the patient's condition.

- Big Data analysis service: The Big Data analysis techniques on data stored in the cloud layer enable the valid data to be used for various purposes such as predicting areas infected with COVID-19 in the coming days based on the map, predicting the number of infected patients, the survival rate of patients, and recurrence of the disease in discharged patients. Users of this service are managers at various levels of decision-making and healthcare professionals.

- Tele-multiple disciplinary care service: This service allows the exchange of views and experiences of physicians in different care centers regarding patients with COVID-19. This service provides multidisciplinary care for patients.

- Teleradiology service: This service is a channel for the exchange of the chest CT scan between radiologists in different national and international locations, which provides a valid interpretation of complex chest examinations.

- Radiological image processing service: This service provides intelligent analysis of the chest CT scan. Using image processing techniques and deep learning on the chest CT images archived on the cloud layer( in PACS), the service can be used as a smart digital assistant for medical staff to diagnose COVID-19 disease.

- Tele-prescription service: This service is used to provide drug prescription services to quarantine patients at home by physicians. After prescribing the drug, the drug is automatically registered in the pharmacy system and can be collected by patients.

- Tracking service: Traffic routes are being tracked via mobile phone GPS in recent days for all people who have been diagnosed with COVID-19 disease. Using this service, different areas can be classified in terms of risk level.

- Fog update service: This service is responsible for adding parameters and new risk factors, and editing the value range of specified parameters in order to determine the risk threshold. When a new parameter needs to be added by experts, the process of defining the parameter and determining its normal range will take place on the cloud layer. This new information must also be applied to the fog nodes for use. It is the job of fog update service to do the process of updating the fog nodes.

\section{Discussion:}

Table 2 shows the layers and services available in some studies related to coronavirus in recent years, as well as the proposed framework, such as cloud layer (CC), fog layer (FC), and internet of things (IoT). The services include; Big data (BD), Decision Making(DM), priority queue (PQ), Telepsychological(TP), Tele-multiple disciplinary care ( TMDC), Tele-homecare monitoring (THCM), Tele-hospital monitoring (THM), E-Self-assessment (ESA), Tele-prescription(TP), Tele-radiology (TR), Patient tracking (PT), Radiological image processing (RIP), Tele-information(TI) and Tele-training services (TT).

Table 2

Related work of Corona Virus healthcare framework based on ICT services

\begin{tabular}{|c|c|c|c|c|c|c|c|c|c|c|c|c|c|c|c|c|c|c|}
\hline Study & $\begin{array}{l}\text { Application } \\
\text { domain }\end{array}$ & IOT & FC & $\mathrm{CC}$ & $P Q$ & DM & BD & TP & TMDC & THCM & THM & ESA & TP & TR & PT & RIP & $\mathrm{TI}$ & $\mathrm{TN}$ \\
\hline $\begin{array}{l}\text { Sandhu et } \\
\text { al. [49] }\end{array}$ & $\begin{array}{l}\text { MERS-COV } \\
\text { prediction } \\
\text { model }\end{array}$ & प & $x$ & $\square$ & $x$ & 口 & प & $x$ & $x$ & $x$ & $x$ & प & $x$ & $x$ & प & $x$ & $x$ & $x$ \\
\hline \multirow{2}{*}{$\begin{array}{l}\text { Amir H. } \\
\text { avarpanah } \\
\text { [42] }\end{array}$} & COVID-19 & $x$ & $x$ & $x$ & $x$ & $x$ & $x$ & $x$ & $x$ & $x$ & $x$ & $x$ & $x$ & $\square$ & $x$ & $x$ & $x$ & $x$ \\
\hline & $\begin{array}{l}\text { Teleconsultation } \\
\text { Service } \\
\text { (WhatsApp } \\
\text { Messenger } \\
\text { chanel) }\end{array}$ & & & & & & & & & & & & & & & & & \\
\hline $\begin{array}{l}\text { Raghav RS. } \\
\text { et al[78] }\end{array}$ & $\begin{array}{l}\text { prevention of } \\
\text { SARS disease }\end{array}$ & 口 & 口 & प & $x$ & 口 & प & 口 & 口 & 口 & प & प & $x$ & $x$ & प & $x$ & $x$ & $x$ \\
\hline $\begin{array}{l}\text { Li Bai ,et } \\
\text { al[28] }\end{array}$ & $\begin{array}{l}\text { COVID-19 } \\
\text { Intelligent } \\
\text { Diagnosis and } \\
\text { Treatment } \\
\text { Assistant } \\
\text { Program } \\
\text { (nCapp) }\end{array}$ & प & $x$ & 口 & $x$ & 口 & प & $x$ & प & 口 & $x$ & प & $x$ & $x$ & 口 & $x$ & प & प \\
\hline \multirow{2}{*}{$\begin{array}{l}\text { Proposed } \\
\text { architecture }\end{array}$} & COVID-19 & $\square$ & $\square$ & प & $\square$ & $\square$ & प & $\square$ & $\square$ & प & प & $\square$ & प & प & प & प & प & प \\
\hline & $\begin{array}{l}\text { Conceptual } \\
\text { model for } \\
\text { management } \\
\text { and control } \\
\text { outbreak }\end{array}$ & & & & & & & & & & & & & & & & & \\
\hline
\end{tabular}


Studies that have been conducted on infectious diseases by information technology services has benefited from these services but these services are not integrated and a comprehensive architecture for communicable diseases such as coronavirus has not yet been developed. The innovation that can be mentioned in this research is the use of priority queue service as a service of the fog layer. This service is necessary because risk factors can play an important role in the recovery or death of people with coronavirus [79]. By taking advantage of the priority queue service and prioritizing affected people based on the importance of risk factors identified for this virus, faster treatment will be provided for this group of patients (with at least one risk factor) in critical situations and irreparable consequences will be prevented.

The proposed model has different aspects such as diagnosis, prevention, training and information, which together provide the ability to manage and control the prevalence of infectious disease. Diagnostic issues create challenges for health systems during quarantine. For example, the accuracy of CT diagnosis of COVID-19 infection depends on the skill of radiologist. The limited number of trained chest radiologists specifically for this disease is a challenge and an obstacle to a valid interpretation of complex chest examinations [42]. Recent events, such as the global outbreak of COVID-19, highlight the need for rapid and effective tools for recording, archiving, transmitting and interpreting radiological images of patients with pneumonia [45]. Teleradiology and the processing of radiological images using machine learning techniques are among the services offered in the proposed model that provide accurate and rapid diagnosis of suspected COVID-19 cases. Participants in the survey confirmed the need for both services (with agreement rate of $98 \%$ and $80 \%$, respectively). On the other hand, the existence of multidisciplinary treatment team that includes specialists from different clinical disciplines provides a comprehensive assessment of patients' conditions. In infectious epidemics such as COVID-19, to reduce the accumulations of healthcare staff and subsequent cessation of possible transmission of infection among them, Tele-experience is an efficient service. According to the survey, the amount of agreement with Tele-multiple disciplinary care service was $93.6 \%$. Computer science has been widely used to analyze viral epidemic and examine its changes [4]. The sudden increase in COVID-19 cases has put a lot of pressure on health care services around the world. At the present stage, rapid, accurate, and clinical evaluation of the severity of disease is critical. It is helpful to use machine learning methods to support logistics decision-making and planning in healthcare systems [80]. Predicting patient survival, identifying high-risk patients, and placing them on the treatment priority list will reduce mortality rate [81]. Big data analysis services and data mining are important for both patients' prognosis and controlling the epidemic. Given the importance of stress management in coronavirus epidemic, the use of specialists in the field of Tele-psychological greatly helps to reduce the anxiety of society. On the one hand, families with one infected family member need psychological counseling to reduce their anxiety. They also need to receive advice on special care during quarantine. In order to respond to the psychological pressures of the medical staff in the hospitals that care for patients with the new coronavirus, psychological counseling is a vital measure that should be provided for medical staff [44]. Tele-psychological for medical staff, quarantine people at home, and patients are among the services offered in the proposed model after the approval of participants in the survey. In times of epidemics, using methods that reduce unnecessary referrals to healthcare centers will reduce the risk of infection for health care workers and prevent the spread of disease among healthy people. E-Self-assessment, tele-monitoring (hospital quarantine and home quarantine), and tele-prescription are effective in achieving these goals. The agreement rate with these services was $97.2 \%, 100 \%, 90.4 \%$ and $100 \%$, respectively. Another aspect of the proposed model is education and information. Due to the great concern of people at the outbreak of infectious diseases, the demand for information about the disease will increase, and the general public has almost nothing to do but search for information about the infectious diseases and how to protect themselves against them. In such situations, the internet and social networks provide a platform for the dissemination of false information and rumors. An online study of the spread of online information, the fear, and the risk of infection in acute respiratory syndrome in the Middle East showed that among the online documents examined, negative emotions accounted for $80 \%$ of all posts across the network channels. Therefore, if a new and highly contagious disease such as Covid-19 spreads, the use of a response system to provide reliable information and training content along with medical services will prevent the dissemination of incorrect information in the internet. The participants in this study expressed their agreements on two tele-information and tele training services with the agreement rate of $100 \%$. In the proposed model, the need for using tracking service to prevent the spread of new coronavirus was confirmed with the agreement rate of $100 \%$. This service is important to distinguish the population at risk using GPS.

\section{Conclusion:}

The most important part of health care is the continuous monitoring of the spread of any fatal disease. Looking at the future of prevention and control of epidemics, it seems that the existence of a comprehensive e-health infrastructure along with reducing public exposure to infected people facilitate the rapid and appropriate use of treatment. Therefore, these infrastructures can play an important role in dealing with emergencies and the widespread of diseases. Of course, every technology has its own challenges, and for e-health, access to the right internet speed and bandwidth is a challenge. The use of e-health infrastructure in epidemic conditions has great potential in improving epidemiological research, disease control and clinical management. It seems that developing societies should take a major step in setting up a comprehensive infrastructure for managing, preventing, predicting, and controlling communicable diseases so that, they can benefit from e-health services at the beginning of these outbreaks. Studies conducted since the outbreak of COVID-19 have examined the latest information on epidemiology, clinical features, diagnosis, treatment and prevention of this infection, and this study is the first study that has proposed a comprehensive conceptual model of e-health regarding this disease.

A conceptual model has been proposed in this study by reviewing the texts, and using the technical experiences of research team and the opinions of experts in various fields. Researchers in future studies should plan to develop a comprehensive national software platform based on the proposed model in this study.

\section{Abbreviations:}

ICT: Information and Communication Technology; IOT: Internet of Things; SARS: severe acute respiratory syndrome; CoV: Corona viruses; MERS: Middle East Respiratory Syndrome; BBN: Bayesian Belief Networks; SVM: Support Vector Machine; K-NN: K-Nearest Neighbor; GIS: geographic information 
system; SNA: Social Network Analysis; RFID: radio frequency identification; PACS: Picture Archiving and Communication Systems; CC: cloud layer; FC: fog layer; BD: Big data; DM: Decision Making; PQ: priority queue; TPS:Tele-psychological; TMDC: Tele-multiple disciplinary care; THCM: Tele-homecare monitoring; THM: Tele-hospital monitoring; ESA: E-Self-assessment; TP: Tele-prescription; TR: Tele-radiology; PT: Patient tracking; RIP: Radiological image processing; TI: Tele-information; TT: Tele-training services;

\section{Declarations:}

Acknowledgements: Not applicable.

Authors 'contributions: all authors have read and approved the manuscript. AY, RS, RR, SFD, MS, YG, MZ contributed equally to the study design. AZ and MZ prepared the manuscript and MS and SFD revised it critically. AZ, SFD, MS AND MZ was the initiator of the literature search and review, reading, categorizing and analyzing and AZ drew the proposed model. RS and RR AND YG additionally performed supervisory tasks.

Funding Information: Shiraz University of Medical Sciences,(grant number: 99-01-07-22416).

Availability of data and materials: Not applicable.

Ethical approval and consent: This article is taken from the research entitled " COVID-19 and Information Communication Technology: Common elements in an integrative framework for Controlling, Prevention, prediction, and Monitoring of New Corona Viruses Disease" Evaluated by: Shiraz University of Medical Sciences, Approval Date: 2020-07-22; Approval ID: IR.SUMS.REC.1399.558 URL: https://ethics.research.ac.ir/ProposalCertificateEn.php? id=144441\&Print=true\&NoPrintHeader=true\&NoPrintFooter=true\&NoPrintPageBorder=true\&LetterPrint=true

Consent for publication: Not applicable.

Consent to participate: Informed consent was obtained from all individual participants included in the study.

Competing interests: The authors declare that they have no competing interests.

\section{References:}

1. Singh, S., et al., Fog computing and loT based healthcare support service for dengue fever. International Journal of Pervasive Computing and Communications, 2018.

2. Wang, X., et al., Enabling smart personalized healthcare: a hybrid mobile-cloud approach for ECG telemonitoring. IEEE journal of biomedical and health informatics, 2013. 18(3): p. 739-745.

3. Organization, W.H. Managing epidemics:Key facts about major deadly diseases. 2018; Available from: https://www.who.int/emergencies/diseases/managing-epidemics/en/.

4. Sandhu, R., H.K. Gill, and S.K. Sood, Smart monitoring and controlling of Pandemic Influenza A (H1N1) using Social Network Analysis and cloud computing. Journal of Computational Science, 2016. 12: p. 11-22.

5. Shin, H.C., et al., Healthcare utilization monitoring system in Korea. Healthcare informatics research, 2015. 21(3): p. 184-190.

6. Milinovich, G.J., et al., Internet-based surveillance systems for monitoring emerging infectious diseases. The Lancet infectious diseases, 2014. 14(2): p. $160-168$.

7. Rothberg, M.B. and K.A. Martinez, Influenza Management via Direct to Consumer Telemedicine: an Observational Study. Journal of General Internal Medicine, 2020: p. 1-3.

8. de Groot, R.J., et al., Commentary: Middle East respiratory syndrome coronavirus (MERS-CoV): announcement of the Coronavirus Study Group. Journal of virology, 2013. 87(14): p. 7790-7792.

9. Warwick, M., et al., Self-management and symptom monitoring among older adults with chronic obstructive pulmonary disease. Journal of advanced nursing, 2010. 66(4): p. 784-793.

10. Ohannessian, R., Telemedicine: Potential applications in epidemic situations. European Research in Telemedicine/La Recherche Européenne en Télémédecine, 2015. 4(3): p. 95-98.

11. Milinovich, G.J., R.J.S. Magalhães, and W. Hu, Role of big data in the early detection of Ebola and other emerging infectious diseases. The Lancet Global Health, 2015. 3(1): p. e20-e21.

12. WHO.

13. Lu, H., C.W. Stratton, and Y.W. Tang, Outbreak of Pneumonia of Unknown Etiology in Wuhan China: the Mystery and the Miracle. Journal of Medical Virology.

14. Wu, Z. and J.M. McGoogan, Characteristics of and important lessons from the coronavirus disease 2019 (COVID-19) outbreak in China: summary of a report of 72314 cases from the Chinese Center for Disease Control and Prevention. Jama, 2020.

15. Rothan, H.A. and S.N. Byrareddy, The epidemiology and pathogenesis of coronavirus disease (COVID-19) outbreak. Journal of Autoimmunity, 2020: p. 102433

16. Sun, P., et al., Understanding of COVID-19 based on current evidence. Journal of medical virology, 2020.

Page $11 / 15$ 
17. Novel, C.P.E.R.E., The epidemiological characteristics of an outbreak of 2019 novel coronavirus diseases (COVID-19) in China. Zhonghua liu xing bing xue za zhi= Zhonghua liuxingbingxue zazhi, 2020. 41(2): p. 145.

18. Li, Y. and L. Xia, Coronavirus Disease 2019 (COVID-19): Role of chest CT in diagnosis and management. American Journal of Roentgenology, 2020: p. 1-7.

19. Kooraki, S., et al., Coronavirus (COVID-19) outbreak: what the department of radiology should know. Journal of the American college of radiology, 2020 .

20. Zu, Z.Y., et al., Coronavirus disease 2019 (COVID-19): A perspective from China. Radiology, 2020: p. 200490.

21. Bernheim, A., et al., Chest CT findings in coronavirus disease-19 (COVID-19): relationship to duration of infection. Radiology, 2020 : p. 200463.

22. Gao, J., Z. Tian, and X. Yang, Breakthrough: Chloroquine phosphate has shown apparent efficacy in treatment of COVID-19 associated pneumonia in clinical studies. Bioscience trends, 2020.

23. Zhang, L. and Y. Liu, Potential interventions for novel coronavirus in China: A systematic review. Journal of medical virology, 2020.

24. Peeri, N.C., et al., The SARS, MERS and novel coronavirus (COVID-19) epidemics, the newest and biggest global health threats: what lessons have we learned? International journal of epidemiology, 2020.

25. Rasmussen, S.A., et al., Coronavirus Disease 2019 (COVID-19) and Pregnancy: What obstetricians need to know. American journal of obstetrics and gynecology, 2020.

26. Zhai, Y., et al., From Isolation to Coordination: How Can Telemedicine Help Combat the COVID-19 Outbreak? medRxiv, 2020.

27. Jahanbin, K. and V. Rahmanian, Using twitter and web news mining to predict COVID-19 outbreak. 2020, Medknow Publications.

28. Bai, L., et al., Chinese experts' consensus on the Internet of Things-aided diagnosis and treatment of coronavirus disease 2019 . Clinical eHealth, 2020.

29. Pawar, P., et al., A framework for the comparison of mobile patient monitoring systems. Journal of biomedical informatics, 2012. 45(3): p. 544-556.

30. Yazdani, A., et al., Scalable architecture for telemonitoring chronic diseases in order to support the CDSSs in a common platform. Acta Informatica Medica, 2018. 26(3): p. 195

31. Angelucci, A. and A. Aliverti, Telemonitoring systems for respiratory patients: technological aspects. Pulmonology, 2020.

32. Ward, M.M., M. Jaana, and N. Natafgi, Systematic review of telemedicine applications in emergency rooms. International journal of medical informatics, 2015. 84(9): p. 601-616.

33. Chauhan, V., et al., Novel coronavirus (COVID-19): Leveraging telemedicine to optimize care while minimizing exposures and viral transmission. Journal of Emergencies, Trauma, and Shock, 2020. 13(1): p. 20.

34. Rivers, C.M., et al., Modeling the impact of interventions on an epidemic of Ebola in Sierra Leone and Liberia. PLoS currents, 2014. 6.

35. Olu, O.O., et al., Contact tracing during an outbreak of Ebola virus disease in the Western Area districts of Sierra Leone: lessons for future Ebola outbreak response. Frontiers in public health, 2016. 4: p. 130.

36. Tom-Aba, D., et al., Innovative technological approach to Ebola virus disease outbreak response in Nigeria using the open data kit and form hub technology. Plos one, 2015. 10(6).

37. Tracey, L., et al., EbolaTracks: an automated SMS system for monitoring persons potentially exposed to Ebola virus disease. Eurosurveillance, 2015. 20(1): p. 20999.

38. Control, C.f.D. and Prevention, Fact sheet: basic information about SARS. CDC, Editor. January, 2004. 13.

39. Organization, W.H., Human infection with influenza A (H7N9) virus in China-update. Geneva, Switzerland: WHO Available: http://www. who. int/influenza/human_animal_interface/influenza_h7n9/08_ReportWebH7N9Number. pdf. Accessed, 2013.

40. Control, C.f.D. and Prevention, Preparing for Ebola: What US hospitals can learn from Emory Healthcare and Nebraska Medical Center. 2014.

41. Kang, L., et al., The mental health of medical workers in Wuhan, China dealing with the 2019 novel coronavirus. The Lancet Psychiatry, 2020. 7(3): p. e14.

42. Davarpanah, A.H., et al., Novel Screening and Triage Strategy in Iran During Deadly Coronavirus Disease 2019 (COVID-19) Epidemic: Value of Humanitarian Teleconsultation Service. J Am Coll Radiol, 2020.

43. Wu, P., et al., The psychological impact of the SARS epidemic on hospital employees in China: exposure, risk perception, and altruistic acceptance of risk. Can J Psychiatry, 2009. 54(5): p. 302-11.

44. Chen, Q., et al., Mental health care for medical staff in China during the COVID-19 outbreak. Lancet Psychiatry, 2020. 7(4): p. e15-e16.

45. Javadi, M., et al., Diagnosing pneumonia in rural Thailand: Digital cameras versus film digitizers for chest radiograph teleradiology. Int J Infect Dis, 2006. 10(2): p. 129-35.

46. Li, Y. and L. Xia, Coronavirus Disease 2019 (COVID-19): Role of Chest CT in Diagnosis and Management. AJR Am J Roentgenol, 2020 : p. 1-7.

47. Al-Turaiki, I., M. Alshahrani, and T. Almutairi, Building predictive models for MERS-CoV infections using data mining techniques. J Infect Public Health, 2016. 9(6): p. 744-748.

48. AlMoammar, A., L. AlHenaki, and H. Kurdi. Selecting Accurate Classifier Models for a MERS-CoV Dataset. in Proceedings of SAl Intelligent Systems Conference. 2018. Springer.

49. Sandhu, R., S.K. Sood, and G. Kaur, An intelligent system for predicting and preventing MERS-CoV infection outbreak. The Journal of Supercomputing, 2016. 72(8): p. 3033-3056. 
50. Jang, S., et al. Comparison between SARS CoV and MERS CoV Using Apriori Algorithm, Decision Tree, SVM. in MATEC Web of Conferences. 2016. EDP Sciences.

51. Kurdia, H. and N. AlMansour. Identifying accurate classifier models for a text-based MERS-CoV dataset. in 2017 Intelligent Systems Conference (IntelliSys). 2017. IEEE.

52. Kerdprasop, N. and K. Kerdprasop. Remote sensing based modeling of dengue outbreak with regression and binning classification. in 2016 2nd IEEE International Conference on Computer and Communications (ICCC). 2016. IEEE.

53. Lai, P.-C., et al., An early warning system for detecting H1N1 disease outbreak-a spatio-temporal approach. International Journal of Geographical Information Science, 2015. 29(7): p. 1251-1268.

54. Dias, C. and E. Arruda. Computational Cost Optimization for Influenza A (H1N1) Epidemic Model. in Proceedings of the 7th International Congress on Environmental Modelling and Software, June. 2014.

55. Farah, M., et al., Bayesian emulation and calibration of a dynamic epidemic model for A/H1N1 influenza. Journal of the American Statistical Association, 2014. 109(508): p. 1398-1411.

56. Bello-Orgaz, G., J. Hernandez-Castro, and D. Camacho, A survey of social web mining applications for disease outbreak detection, in Intelligent Distributed Computing VIII. 2015, Springer. p. 345-356.

57. Mykhalovskiy, E. and L. Weir, The global public health intelligence network and early warning outbreak detection. Canadian journal of public health, 2006. 97(1): p. 42-44.

58. Collier, N., et al., BioCaster: detecting public health rumors with a Web-based text mining system. Bioinformatics, 2008. 24(24): p. $2940-2941$.

59. Brownstein, J.S., et al., Surveillance Sans Frontieres: Internet-based emerging infectious disease intelligence and the HealthMap project. PLoS medicine, 2008. 5(7).

60. Keller, M., et al., Use of unstructured event-based reports for global infectious disease surveillance. Emerging infectious diseases, $2009.15(5)$ : p. 689.

61. Linge, J.P., et al., MedISys: medical information system, in Advanced ICTs for disaster management and threat detection: Collaborative and distributed frameworks. 2010, IGI Global. p. 131-142.

62. Carneiro, H.A. and E. Mylonakis, Google trends: a web-based tool for real-time surveillance of disease outbreaks. Clinical infectious diseases, 2009. 49(10): p. 1557-1564.

63. Ritterman, J., M. Osborne, and E. Klein. Using prediction markets and Twitter to predict a swine flu pandemic. in 1st international workshop on mining social media. 2009.

64. Culotta, A. Towards detecting influenza epidemics by analyzing Twitter messages. in Proceedings of the first workshop on social media analytics. 2010.

65. Aramaki, E., S. Maskawa, and M. Morita. Twitter catches the flu: detecting influenza epidemics using Twitter. in Proceedings of the conference on empirical methods in natural language processing. 2011. Association for Computational Linguistics.

66. Lee, K., A. Agrawal, and A. Choudhary. Real-time disease surveillance using twitter data: demonstration on flu and cancer. in Proceedings of the 19th ACM SIGKDD international conference on Knowledge discovery and data mining. 2013.

67. Bodnar, T. and M. Salathé. Validating models for disease detection using twitter. in Proceedings of the 22nd International Conference on World Wide Web. 2013.

68. Aceto, G., V. Persico, and A. Pescapé, Industry 4.0 and Health: Internet of Things, Big Data, and Cloud Computing for Healthcare 4.0. Journal of Industrial Information Integration, 2020: p. 100129.

69. Gubbi, J., et al., Internet of Things (IOT): A vision, architectural elements, and future directions. Future generation computer systems, 2013. 29(7): p. $1645-1660$.

70. Botta, A., et al., Integration of cloud computing and internet of things: a survey. Future Generation Computer Systems, 2016. 56: p. 684-700.

71. Song, Y., et al., Prospect and application of Internet of Things technology for prevention of SARIs. Clinical eHealth, 2020.

72. Zhu, H., et al., IOT PCR for pandemic disease detection and its spread monitoring. Sensors and Actuators B: Chemical, 2020. 303: p. 127098.

73. Yi, S., C. Li, and Q. Li. A survey of fog computing: concepts, applications and issues. in Proceedings of the 2015 workshop on mobile big data. 2015. ACM.

74. Firdhous, M., O. Ghazali, and S. Hassan. Fog computing: Will it be the future of cloud computing? 2014. The Third International Conference on Informatics \& Applications (ICIA2014).

75. Sood, S.K. and I. Mahajan, Wearable loT sensor based healthcare system for identifying and controlling chikungunya virus. Computers in Industry, 2017. 91: p. 33-44.

76. Tazkia, R.A.K., V. Narita, and A.S. Nugroho. Dengue outbreak prediction for GIS based Early Warning System. in 2015 International Conference on Science in Information Technology (ICSITech). 2015. IEEE.

77. Sareen, S., S.K. Sood, and S.K. Gupta, IoT-based cloud framework to control Ebola virus outbreak. Journal of Ambient Intelligence and Humanized Computing, 2018. 9(3): p. 459-476.

78. Raghav, R. and P. Dhavachelvan, Bigdata fog based cyber physical system for classifying, identifying and prevention of SARS disease. Journal of Intelligent \& Fuzzy Systems, 2019. 36(5): p. 4361-4373. 
79. Liu, W., et al., Analysis of factors associated with disease outcomes in hospitalized patients with 2019 novel coronavirus disease. Chinese medical journal, 2020.

80. Yazdani, A., et al., Diagnosis of Breast Cancer Using Decision Tree, Artificial Neural Network and Naive Bayes to Provide a Native Model for Fars Province. Journal of Payavard Salamat, 2019. 13(3): p. 0-0.

81. Zhou, C., et al., COVID-19: challenges to GIS with big data. Geography and Sustainability, 2020.

\section{Figures}

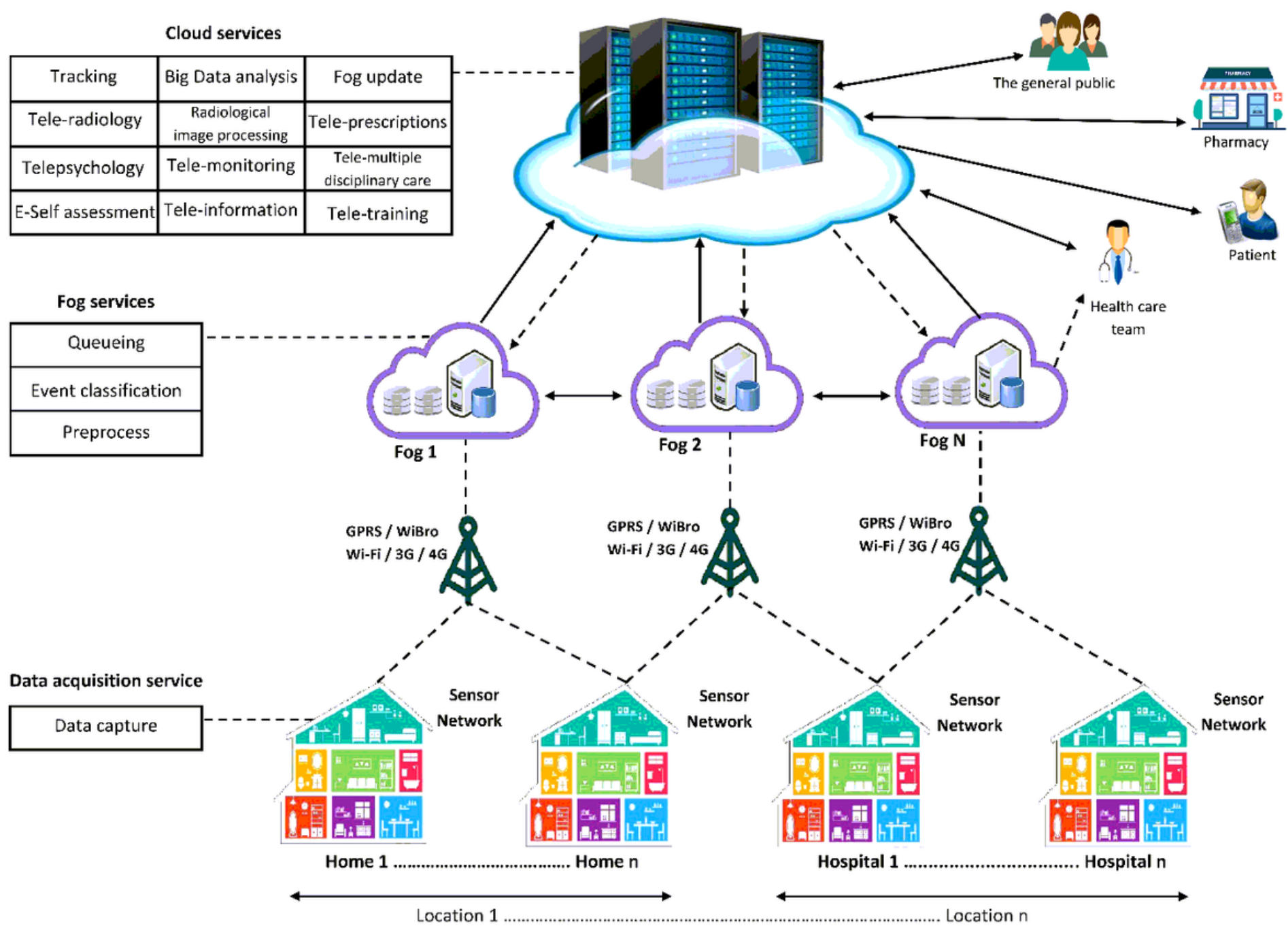

Figure 1

Proposed conceptual model 
Data acquisition layer

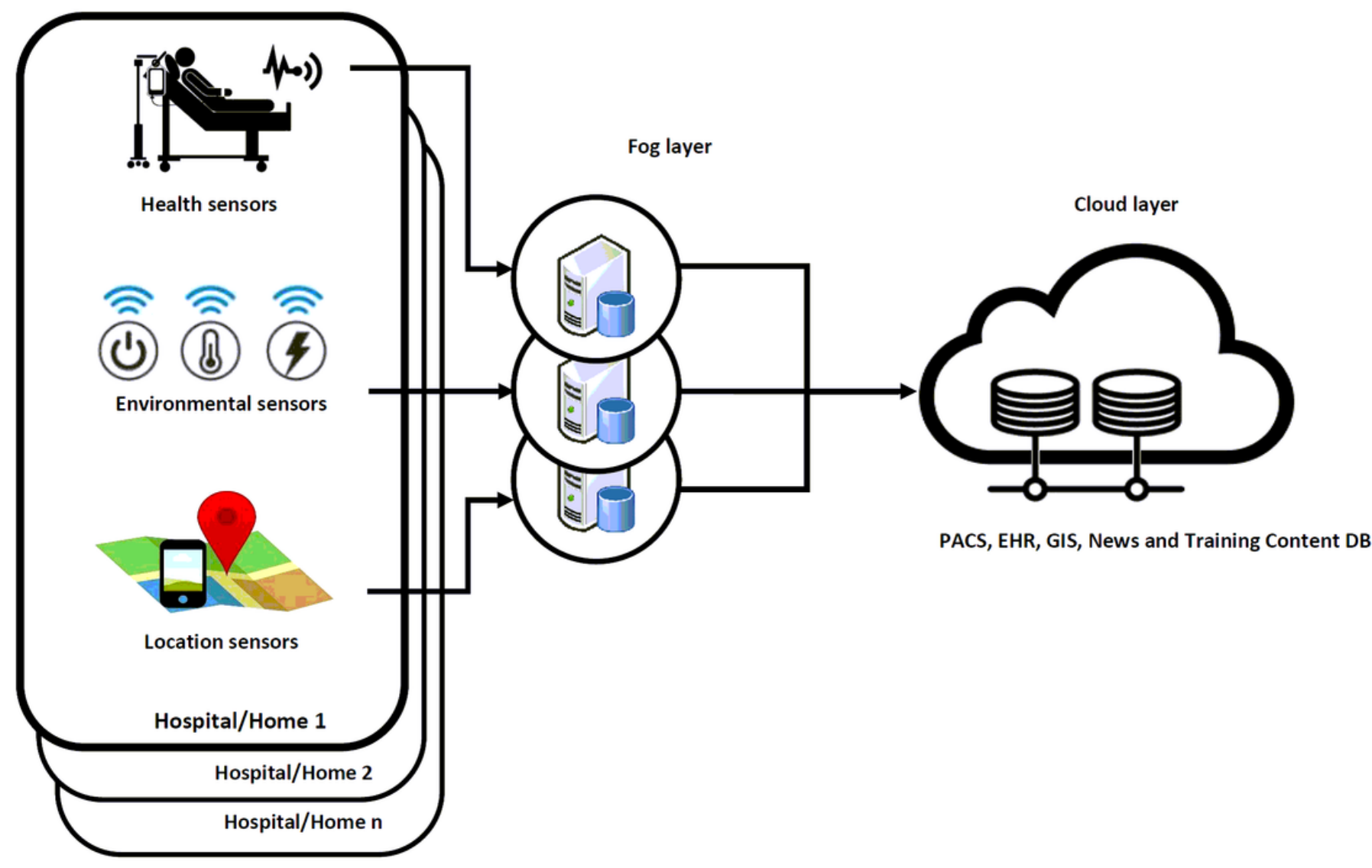

Figure 2

Three layers model

Fog Nodes

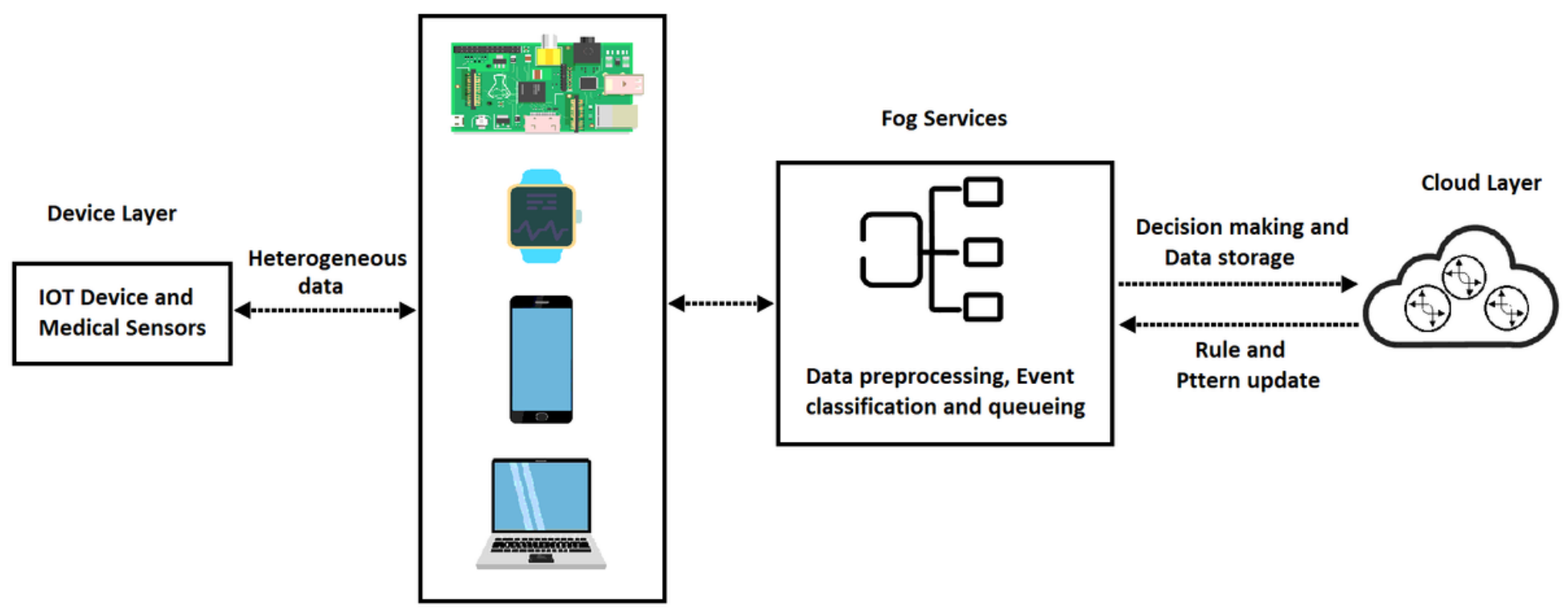

Figure 3

The fog layer details 